\title{
Circuit
}

Musiques contemporaines

\section{Les auteurs}

Volume 14, numéro 2, 2004

\section{Montréal/Nouvelles Musiques}

URI : https://id.erudit.org/iderudit/902321ar

DOI : https://doi.org/10.7202/902321ar

Aller au sommaire du numéro

\section{Éditeur(s)}

Les Presses de l'Université de Montréal

ISSN

1183-1693 (imprimé)

1488-9692 (numérique)

Découvrir la revue

Citer ce document

(2004). Les auteurs. Circuit, 14(2), 125-126. https://doi.org/10.7202/902321ar

Ce document est protégé par la loi sur le droit d'auteur. L'utilisation des services d'Érudit (y compris la reproduction) est assujettie à sa politique d'utilisation que vous pouvez consulter en ligne.

https://apropos.erudit.org/fr/usagers/politique-dutilisation/ 


\section{LES AUTEURS}

\section{Réjean Beaucage}

Réjean Beaucage est rédacteur adjoint au magazine montréalais La Scena Musicale et chroniqueur en musique classique à l'hebdomadaire Voir. Il collabore également à la revue Improjazz (France).

\section{Philip Blackburn}

Depuis 1991, Philip Blackburn est directeur de l'American Composers Forum. Grand spécialiste de l'œuvre de Harry Partch, il a travaillé pendant 15 années à l'écriture d'Enclosures, ouvrage qui s'est mérité le ASCAP Deems Taylor Award, ainsi qu'à la création de plusieurs disques compacts et vidéos portant sur la vie et l'œuvre du compositeur. Il a complété un doctorat en composition à l'Université d'lowa, où il a étudié avec Kenneth Gaburo. Il dirige aujourd'hui la maison de disques innova Recordings newmusic et est cofondateur du Sonic Circuits International Electronic Music Festival.

\section{Ariane Couture}

Après l'obtention d'un DEC en musique au cégep de Saint-Laurent en 2001, Ariane Couture poursuit des études de baccalauréat en musicologie à I'Université de Montréal. Elle est membre du Cercle des étudiants en composition de l'Université de Montréal $(\mathrm{CeCo})$, pour lequel elle est responsable de la rédaction du $\mathrm{CeCo}$ Hebdo, et membrefondateur du Cercle de musicologie. Elle a récemment remporté une bourse du Fonds Marywonne Kendergi pour la recherche sur la musique canadienne et québécoise, ce qui lui permeltra d'entreprendre dès cet automne des études de deuxième cycle en musicologie.

\section{Andrew Culver}

En tant que compositeur, Andrew Culver a écrit pour des formations de chambre et orchestrales, et produit de la musique électronique et informatique. Il a également fait de la sculpture, du cinéma, de l'éclairage, des installations et dirigé un opéra. Il a passé de nombreuses années à inventer des instruments et à en jouer. Il a développé des procédés et des logiciels de composition qui emploient souvent des systèmes structuraux et proportionnels et (ou) des opérations de hasard. II a signé des textes sur la musique, l'art et l'anarchie. De 1981 à 1992, il a travaillé quotidiennement avec John Cage. En collaboration avec Frank Scheffer en 1985, il a fait From Zero, quatre films sur Cage récemment parus sur DVD (Mode Records, New York). L'adresse de son site personnel est : unw.anarchicharmony.org.

\section{Yannick Delbecque}

Après une maîtrise en mathématiques fondamentales à l'Université de Montréal, Yannick Delbecque a entrepris un doctorat en informatique théorique au School of Computer Science de l'Université McGill. II s'intéresse à la théorie des jeux comme outil pour l'analyse de la causalité en logique, en sémantique des langages de programmation et en informatique quantique.

Du côté musical, après des études collégiales en musique, Yannick Delbecque a mené une vie d'autodidacte parsemée de quelques cours d'analyse et d'écriture à la Faculté de musique de l'Université de Montréal. Il a aussi participé au camp de composition musicale du Domaine Forget en 1998. II travaille présentement à un projet de pièce pour orchestre et bande, ef à une application de ses idées sur la causalité et la perception sonore en analyse musicale.

\section{Michel Duchesneau}

Professeur à la Faculté de musique de I'Université de Montréal, Michel Duchesneau a complété un doctorat en musicologie à l'Université Laval, puis il a poursuivi des recherches postdoctorales en France. Auteur de plusieurs articles el conférences sur la musique française de la première moitié du $x x^{e}$ siècle, il a aussi publié un livre intitulé L'avantgarde musicale en France et ses sociétés de 1871 à 1939 (Mardaga, 1997). Outre ses activités de musicologue, Michel Duchesneau s'intéresse à la gestion dans le domaine des arts et a entre autres occupé le poste de directeur général de la Société de musique contemporaine du Québec de 1997 à 2002. Il enseigne aujourd'hui le cours de gestion de carrière à la Faculté de musique de l'Université de Montréal. Rédacteur en chef de la revue Circuit, musiques contemporaines depuis 2000, son intérêt pour l'étude des courants musicaux d'avant-garde l'a poussé à fonder en 2003 l'Observatoire international de la création musicale $(\mathrm{O} \mid \mathrm{CM})$, équipe de recherche interdisciplinaire qui se consacre à l'étude de la création musicale de la fin du XIX siècle jusqu'à nos jours.

\section{Nicolas Gilbert}

Nicolas Gilbert est un jeune compositeur né à Montréal en 1979. Ses œuvres ont été commandées et interprétées par plusieurs solistes, ensembles et organismes canadiens et étrangers. Au cours des dernières années, il a reçu plusieurs bourses du Conseil des arts et des lettres du 
Québec, du Conseil des Arts du Canada et du Fonds Québécois de Recherche sur la Société et la Culture. Il est actuellement compositeur-résident à la Chapelle historique du BonPasteur de Montréal et est également président de la société Codes d'accès, un organisme sans but lucratif fondé en 1985 se consacrant à la diffusion de la musique contemporaine dans des contextes hors-normes. II a fréquenté de nombreux établissements d'enseignement dont le Conservatoire de musique de Montréal, l'Université de Montréal, I'Université Nankai (Tianjin, Chine) et I'Université d'État de Saint-Pétersbourg (Russie). Nicolas Gilbert poursuit actuellement des études de doctorat auprès de John Rea à I'Université McGill.

\section{Olivier Girouard}

Olivier Girouard termine présentement un baccalauréat en écriture musicale et en chant classique à l'Université de Montréal. Depuis 2002, il est membre de la cociété Code d'accès et, depuis septembre 2002, préside le Cercle des étudiants compositeurs de I'Université de Montréal $(\mathrm{CeCo}$ pour lequel il organise divers concerts, ateliers et conférences promouvant l'art contemporain.

\section{Jonathan Goldman}

Après avoir complété des études en philosophie et en mathématiques à I'Université McGill, Jonathan Goldman a obtenu une maîtrise en musicologie de l'Université de Montréal en 1999. II poursuit des études de doctorat en musicologie à la Faculté de musique de l'Université de Montréal, sous la direction de Jean-Jacques Nattiez, dont il est également l'assistant de recherche. II est membre du comité de rédaction de Circuit, musiques contemporaines depuis 1999.

\section{Jean-Dominique Marco}

Depuis 1990, Jean-Dominique Marco est directeur général de Musica, le festival international des musiques d'aujourd'hui à Strasbourg. Il est également président du Réseau Varèse, réseau européen œuvrant pour la création et la diffusion culturelles qui regroupe aujourd'hui 16 structures musicales (festivals, théâtres, etc.) de 11 pays d'Europe. Officier de l'Ordre national du Mérite, Commandeur des Arts et des Lettres, Jean-Dominique Marco est une figure importante du milieu des musiques nouvelles en Europe.

\section{Maxime McKinley}

Maxime McKinley étudie présentement la composition, l'analyse et l'harmonie au Conservatoire de musique du Québec à Montréal, auprès de Michel Gonneville, Serge Provost et Jacques Faubert. Son œuvre pour orchestre Devri Kebir, l'inventeur (2002), une commande de l'Association des orchestres de jeunes du Québec (AOSQ), a été réalisée grâce à une bourse du Conseil des arts et des lettres du Québec. Elle a été entendue au Festival 2003 de l'AOJQ à Laval puis, en France, lors de festivals à Créon et Jonzac. Zénon, l'alchimiste (20012002), pour 16 instruments et récitantténor, s'est mérité un prix lors de l'édition 2003 du Concours des jeunes compositeurs de la Socan. II a prononcé quelques conférences et a participé à la saison 2002-2003 de la société Codes d'accès comme membre du conseil d'administration.

\section{Paul Steenhuisen}

Paul Steenhuisen est un compositeur et un fanatique de l'audio. Sa musique a été jouée et radiodiffusée dans plus de 25 pays. En plus de ses activités de musicien, il élabore présentement une série d'entrevues avec d'autres compositeurs canadiens. II enseigne la composition à l'Université d'Alberta depuis septembre 2003. L'adresse de son site personnel est : http://members.shaw.ca/steenhuisen. 